Pérez Juste, Ramón (2016). ¿Quo vadis, evaluación? Reflexiones pedagógicas en torno a un tema tan manido como relevante. Revista de Investigación Educativa, 34(1), 13-30.

DOI: http://dx.doi.org/10.6018/rie.34.1.239381

\title{
¿Quo vadis, evaluación? Reflexiones pedagógicas en torno a un tema tan manido como relevante
}

\author{
Evaluation, quo vadis? \\ Pedagogical reflections on a trite yet highly relevant subject
}

Ramón Pérez Juste

\begin{abstract}
Resumen
En este artículo se presentan una serie de reflexiones sobre la evaluación desde perspectivas radicalmente pedagógicas en las que el autor pretende analizar las implicaciones que una determinada concepción de la misma, concretada en unos principios básicos, representan para un buen hacer al servicio de la calidad de la educación. El autor defiende un enfoque unitario, armónico y coherente de la evaluación del aprendizaje y de la enseñanza, de los programas y de los centros educativos, extensible a otras manifestaciones como pueden ser los currículos universitarios y los sistemas educativos, aunque estos últimos tengan otro tipo de implicaciones que aquí no corresponde abordar.

Palabras clave: calidad de la educación, educación integral, concepción de la evaluación, evaluación integral.
\end{abstract}

\begin{abstract}
This paper introduces some reflections on evaluation from truly pedagogical points of view, with a special focus on the implications which a particular view may have on the quality of education. The author stands for a unified, harmonious, coherent approach of evaluation of learning and teaching, as well as of programme evaluation and institutional evaluation, which may be applicable to other bodies such as university curricula and educationl systems. The latter, however, may have other implications which will not be addressed in the text.

Keywords: quality of education, well-rounded education, conception of evaluation, integrated evaluation.
\end{abstract}

Correspondencia: Ramón Pérez Juste

RIE, 2016, 34(1), 13-30 


\section{La evaluación y su trascendencia}

La evaluación, a pesar de su carácter meramente instrumental, de acción al servicio de los objetivos de la Educación en sus diferentes ámbitos, elementos, componentes o manifestaciones, se viene configurando como una de las actividades con mayor trascendencia y repercusión en el marco de las preocupaciones por la calidad. Sobre lo primero, baste recordar las consecuencias de algunas de sus manifestaciones tales como las calificaciones escolares, la acreditación del profesorado, el reconocimiento de la valía de los programas, la certificación de los centros educativos o la utilización de los resultados de la evaluación de los sistemas educativos.

Sobre lo segundo, traigamos a nuestra mente su incidencia en la eficacia de las acciones del profesorado, de los líderes pedagógicos o de los responsables de los sistemas educativos. Pero, por si esto fuera poco, tomemos conciencia de un fenómeno más sibilino por su, digamos, naturaleza silenciosa y cuasi subrepticia: la incidencia sobre la calidad de lo pedagógico en todas sus manifestaciones:

- Conformando el verdadero currículo de la Escuela y del aula: lo que se evalúa o no, y cómo se hace, se impone en las preocupaciones de profesores y alumnos sobre lo que oficialmente se pretende tanto en la normativa legal como en las programaciones del profesorado.

- Condicionando fuertemente el curriculum vitae de los profesores, orientando su actividad hacia aquello que se premia al ser considerado como mérito, con la consiguiente postergación de otras funciones y tareas que pueden ser tan o más relevantes personal y socialmente.

- Orientando el contenido del curriculum académico de los niveles educativos en función de evaluaciones externas, como las pruebas de diagnóstico, PISA o similares.

- Condicionando las instituciones educativas a través de la elaboración y publicidad de los ranking.

- Ayudando-y condicionando-a los responsables políticos a orientar sus decisiones ateniéndose a los datos de los informes sobre indicadores de calidad del sistema.

Demasiadas cosas, y muy relevantes, como para merecer una seria reflexión sobre tan importante concepto, una reflexión que, teniendo en cuenta el destinatario de la misma (el lector de RIE), no pretende centrarse en aspectos concretos o prácticos, ni siquiera en cuestiones técnicas relativas a la metodología, sino en las de fondo, en los principios y en sus consecuencias.

Y es que para los otros enfoques ya contamos con relevantes aportaciones, como las de Nevo (1986) al acotar el concepto, o los Estándares para la evaluación de materiales, programas y proyectos, del Joint Commitee (1994) con sus cuatro grandes criterios y sus 30 estándares (Yarbrough, 2011).

De alguna forma, podemos resumir el objeto de esta reflexión en los principios pedagógicos, técnicos y éticos que condicionan una evaluación pedagógica de calidad, esto es, aquella que contribuye decididamente a la mejora de la realidad evaluada, sea esta la persona o su actividad -tanto de alumnos como de profesores o de la comunidad 
educativa-, una organización educativa, para que cumpla con las funciones que le son atribuidas por la sociedad para la formación y capacitación de las sucesivas generaciones o el sistema educativo de un país, a fin de que, como pide UNESCO (1998), resulte pertinente, es decir: responda a las necesidades, demandas y expectativas sociales del aquí y el ahora de cada sistema.

Obviamente, abarcar tanto en el reducido espacio de un artículo sería una insensatez. Por ello, el grueso de las reflexiones tiene que ver con la evaluación del aprendizaje, si bien defiendo un enfoque integral en el que los grandes planteamientos y principios sean de aplicación, también, a la evaluación de la enseñanza y de las organizaciones educativas; $y$, en la medida de lo posible, a los propios sistemas educativos cuando la evaluación se enfoca desde perspectivas puramente pedagógicas.

Claro está que este posicionamiento inicial no desprecia -ni siquiera menospreciaen modo alguno aquellas evaluaciones que inicialmente no se orientan a tal mejora, como ocurre en las sumativas, administrativas (para el ejercicio de la autoridad) o sociopolíticas (para motivar y ganar apoyo público) en terminología de Nevo (1986). Pero sí quiere dejar patente que, en marcos y contextos pedagógicos, aquel es el enfoque fundamental y este solo cobra relevancia por su aportación al primero $\mathrm{y}$, desde otro punto de vista, por el servicio que aquel -el formativo- puede prestar a las evaluaciones sumativas ya que las preventivas (diagnósticas) y formativas se traducen necesariamente en mejoras en los resultados de las sumativas.

\section{Los principios}

Así las cosas, conviene situar nuestro objeto de reflexión en su justo punto; si importante es la evaluación tal como hemos querido evidenciar en el anterior apartado, no podemos dejar tal idea sin matizaciones. La evaluación, por sí sola, no produce efectos positivos, aunque siempre influya; en efecto: en ocasiones, por acción o por omisión, puede dar lugar a consecuencias negativas, a efectos no solo no perseguidos sino no deseados e, incluso, contraproducentes.

Por ello, para que las cosas no sean así, debemos dejar claros algunos principios y planteamientos.

\section{Sentido de la evaluación pedagógica}

Como aspecto previo quiero señalar el sentido de la evaluación, su naturaleza, orientada siempre a la mejora de la realidad evaluada, lo que, como tendremos ocasión de ver, implica varios principios, en particular el de su naturaleza instrumental y su carácter integral, integrado e integrador, sin olvidar su imprescindible dimensión ética.

No podría ser de otra manera ya que, si aceptamos su carácter instrumental, debe servir, por coherencia, a aquello que es la naturaleza de la educación, que no es sino la mejora, el perfeccionamiento integral del ser humano y, por ende, de todo lo que a ello contribuye.

Ante tal planteamiento, la reflexión sobre qué será objeto de evaluación y la especificación de los criterios y las referencias que se aplicarán para su valoración, debidamente hechos públicos entre las partes interesadas, se hace imprescindible. 
Es decir: tanto los objetos a evaluar, como los objetivos a lograr y los medios y recursos a su servicio, habrán de ser intrínsecamente educativos o, al menos, habrán de ser evaluados desde la perspectiva de su contribución a la formación de los educandos.

\section{Los principios. El carácter instrumental}

El primero de los principios, como acabamos de destacar, es el ya enunciado de su carácter instrumental al servicio de los objetos a evaluar y de los objetivos a alcanzar. La evaluación no podrá aportar nada a la calidad de la educación si unos y otros -objetos y objetivos- no responden a las exigencias de la calidad pedagógica. La única forma de plantear algo de relevancia en este aspecto es hacerlo afrontando la evaluación de los mismos, algo que pocas veces se lleva a cabo entre otras razones porque para ello, con frecuencia, al menos en los fundamentales, debemos situarnos fuera incluso de la propia Pedagogía, debiendo analizarlos y valorarlos desde concepciones filosóficas y antropológicas de la persona y de su educación. Esa y no otra es la razón por la que plantearnos la idea misma de "calidad" nos conduce o bien a hacerlo a partir de una concepción filosófico-antropológica del hombre o a enfrentarla al margen de esta, centrándonos en criterios que califico de relativos, como puede ser el planteamiento de De la Orden (1988) al hablar de un sistema de coherencias múltiples:

- Entre fines generales y resultados: Funcionalidad

- Entre fines generales y objetivos, por un lado, y resultados por otro: Eficacia o efectividad

- Entre procesos educativos y medios, por un lado, y resultados, por otro: Eficiencia

Dando por resuelto este tema, algo nada fácil por cierto, la calidad de la evaluación está relacionada con criterios como la coherencia entre sus planteamientos y tales objetos y objetivos, la suficiencia de esta para constatar logros y carencias, la adecuación a los mismos ante la gran diversidad de aquellos y, desde luego, el correcto uso de la información recogida, puesta al servicio de la mejora de la realidad objeto de evaluación y de cuantas personas tengan relación con esa realidad (partes interesadas y afectadas, concepto, a mi juicio, más adecuado que el de "audiencias").

Obviamente, estos criterios de calidad llevan aparejados, como en un racimo, otra serie de consideraciones, ahora claramente técnicas; la coherencia demanda técnicas e instrumentos adecuados, además de válidos y fiables; la suficiencia implica la validez de las medidas y de los datos en general; la adecuación pone en relación la realidad evaluada con las características de los sujetos (validez aparente) y el correcto uso de la información trata de evitar el daño que puede producirse a personas (alumnos, profesores, miembros de la comunidad educativa) y a las organizaciones educativas (centros) cuando se hacen públicos los resultados personalizados que no tienen en sí mismos interés para la mejora del sistema.

Si nos planteamos la evaluación como una actividad de una sola ocasión y descontextualizada del ciclo continuo de la acción educativa, la evaluación poco o nada puede hacer a la hora de fijar y diseñar el currículo de la Escuela y del aula, uno de los objetos de la evaluación. 
En efecto: por lo general, estas actuaciones surgen de planteamientos fundamentalmente políticos y, por ello, con gran frecuencia, ideológicos. Y dado el carácter instrumental de la evaluación, esta podrá contribuir a la constatación del grado de logro y a proveer de información para su futura mejora, pero no para el diseño inicial.

Sin embargo, si, como es deseable, nuestro abordaje lo es en un marco continuo mediante la institucionalización de la evaluación, en un segundo ciclo y en ciclos sucesivos sí podrá avanzarse en la mejora, también, de los propios objetos y objetivos, tanto desde el criterio de personalización, fundamental para la calidad de la educación, como, sobre todo, del de pertinencia -según los planteamientos de UNESCO (1998)forzosamente actualizable y más en estos tiempos en que los cambios sociales son tan profundos, trascendentales y acelerados.

El carácter instrumental puede -y hasta debe- repercutir en aspectos tan importantes como la duración de los procesos evaluativos (no debe consumir más tiempo, energías y recursos que los destinados al logro de los propios objetivos) e, incluso, y precisamente para lograr lo anterior, a planteamientos evaluativos más sencillos, asequibles y eficientes, en los que la metodología aplicable pueda explicar, de modo similar a lo que se pretende con técnicas estadísticas como el análisis factorial, lo más posible con el menor esfuerzo y dedicación, lo que conlleva un notable trabajo de investigación para identificar los componentes esenciales y su debida ponderación.

En efecto: si en el marco de la evaluación del aprendizaje este aspecto no representa una dificultad relevante, salvo en el caso de los profesores que utilizan la evaluación como un instrumento para diferenciar a los alumnos y no para identificar el grado de logro del dominio debidamente definido de aprendizaje, las dificultades se acrecientan cuando de lo que se trata es de evaluar programas o centros. Los modelos de evaluación de programas y de organizaciones educativas son tan ambiciosos que con frecuencia no resultan aplicables si no es consumiendo más tiempo, energía y recursos de los que prudentemente se les puede asignar, y ello en detrimento, precisamente, de las actividades al servicio del logro de lo que la evaluación pretende constatar. Basta conocer los modelos como los de Scriven, Stake o Stufflebeam ${ }^{1}$ y, en alguna medida, el mío propio (2006) para reconocer que su exceso de perfeccionismo en los planteamientos se paga con un consumo excesivo de tiempo y recursos.

Y si de los programas pasamos a los centros educativos, entendidos como organizaciones, la evaluación se sitúa -como alguno de nosotros afirmó en su momento (Escudero, 1980)- en el límite de lo posible. Así, la utilización del Plan EVA (Luján y Puente, 1996) que, según inspectores que lo aplicaron, implicaba a dos evaluadores durante tres meses; de sistemas como $\mathrm{ISO}^{2}, \mathrm{EFQM}^{3}$ o propuestas agrupables bajo el paraguas de Calidad Total, resultan, según algunas de sus concreciones, inaplica-

1 Vid. Stufflebeam, D.L. y Shinkfield, J. (1987). Evaluación sistemática. Guía teórica y práctica. Buenos Aires: Paidós.

2 Vid. Asociación Española para la Calidad (1998). Propuesta para la adaptación de la Norma UNE-EN-ISO 9004-2 a los Servicios educativos y de Formación, Madrid: AEC.

Pérez Juste, R. (Coord.) (2005) Calidad en la Educación, calidad de la Educación. Documentos para una concepción integral e integrada. Madrid: Asociación Española para la Calidad (AEC).

3 Ministerio de Educación y Cultura (1997). Modelo Europeo de Gestión de Calidad, Madrid, MEC; Confederación Española de Centros de Enseñanza (1997). Guía de autoevaluación. Centros Educativos y formativos no universitarios. Modelo europeo de Calidad total en la gestión, Madrid, CECE-Club Gestión de Calidad. 
bles en puridad en muchos casos. Una consecuencia derivada de lo anterior, es la conversión de estos modelos en propuestas en exceso burocráticas y burocratizadas, al servicio no tanto de la mejora de los programas y centros cuanto al logro del reconocimiento social, prostituyéndose la evaluación que pasa de ser un medio al servicio del fin y de los objetivos (la mejora), a convertirse en el fin mismo a lograr (el sello de calidad).

\section{Los principios. Carácter integral, integrado e integrador}

El segundo de los principios, sin que ello signifique valor inferior al primero, es el de su concepción integral, integrada e integradora (Pérez Juste, 2006).

\section{Evaluación integral}

La dimensión integral puede ser analizada desde una doble perspectiva: referida a la evaluación del aprendizaje, o de la enseñanza a su servicio, viene a suponer la necesidad de que todo objeto y todos los objetivos de ambos deban ser potencialmente objeto de evaluación ya que, si así no fuera, estaríamos lanzando el mensaje de que lo no evaluado es de inferior importancia a aquello que sí entra en la evaluación.

Pero referida al hecho pedagógico en general, nos sitúa ante la necesidad de hacer objeto de evaluación no solo lo relativo a la conexión enseñanza/aprendizaje sino todo aquello que contribuye a ella, como son los programas, los centros u organizaciones educativas y hasta los propios sistemas educativos.

Tanto en una como en otra perspectiva, este carácter integral debe evitar no solo reduccionismos y sesgos, sino también perfeccionismos. Y debemos ser conscientes de que estos tipos de errores se dan tanto en la más común de las manifestaciones de la evaluación (aprendizaje) como en el caso de programas, organizaciones y sistemas (PISA, Sistema Estatal de Indicadores de la Educación).

Resalto la expresión "potencialmente", porque la evaluación no puede abarcar todo lo evaluable ya que resultaría inviable por intentar el perfeccionismo; pero sí debe quedar claro que todo lo que es objeto de educación podría constituirse en el objeto real de la evaluación en una ocasión concreta. No olvidemos que, como se ha indicado, la evaluación no debe consumir el tiempo que debe estar dedicado al acto educativo en sí mismo.

Pero también en este punto debemos ser conscientes de las nefastas consecuencias que representan, pedagógicamente hablando, aportaciones como PISA o el Sistema Estatal de Indicadores de la Educación. En el primer caso, su limitación a tres ámbitos de aprendizaje, por destacados que sean, dada la relevancia social que se concede a sus resultados se traduce en un sesgo que perjudica no ya a otros campos académicos sino, más aún, a ámbitos de formación tan relevantes como los relacionados con la inteligencia emocional y otros tipos de inteligencias, siguiendo las ideas de Gardner, sin olvidar otros objetos de enorme trascendencia, como las relaciones humanas, la dimensión moral del ser humano o la estabilidad emocional de los protagonistas, por destacar solo algunos aspectos clave para una educación de calidad. 
Y si pasamos al Sistema Estatal de Indicadores, decisiones tan lamentables como la supresión desde 2010 de los indicadores de procesos educativos, dejan fuera del campo de las decisiones de los responsables educativos todo el ámbito de lo que en verdad preocupa y ocupa a los educadores y a los responsables de los centros. De este modo, la información más relevante para estos queda fuera del sistema de indicadores y se limita a ilustrar las posibles decisiones de los responsables políticos, importante, si, pero muy alejada en sus repercusiones del hecho educativo que tiene lugar en las aulas y en los centros, y, por tanto, de la actividad de sus actores: directivos, alumnos, profesores, comunidad educativa ${ }^{4}$.

\section{Evaluación integrada}

Pero la evaluación, aparte de "integral", ha de ser o, mejor, estar, "integrada". Es verdad que el control de los sistemas, procesos y aprendizajes puede y debe tener su peso para asegurar su funcionamiento y correcto desarrollo. Es verdad que sin la evaluación sumativa podemos o, mejor, estaremos perdidos por falta de una información relevante; es verdad que sin tal planteamiento la necesaria información sobre el sistema presenta carencias fundamentales en un mundo globalizado...

Por tanto, las características propias de una evaluación sumativa deben cuidarse con esmero, con exquisitez, tanto para que rinda sus frutos como para que sus consecuencias se traduzcan en decisiones justas y válidas sobre las personas y organizaciones afectadas.

En efecto: de las calificaciones relativas al aprendizaje o del hacer de los profesores se siguen consecuencias para el alumnado o el propio profesorado que, de no responder a criterios bien fundados y éticamente bien aplicados, pueden dar lugar a situaciones injustas para su promoción, para el logro de recompensas (becas, premios...) con repercusiones graves para su personalidad, afectando a sus sentimientos de valía y autoestima tanto personal como académica y profesional.

Una forma de evitar o, al menos, limitar estos efectos negativos, consiste en integrar la evaluación en el contexto de las actividades ordinarias del profesor, si nos referimos al aprendizaje; actividades que constituyen el programa, entendido como plan de acción al servicio de los objetivos y competencias a cuyo logro se orientan. De este modo, la evaluación, en funciones formativas, se sitúa en el mismo plano que los medios al servicio de aquellos, al igual que los materiales didácticos, la motivación, el diseño de las actividades, la selección de la metodología, los recursos -tecnológicos o no- o la participación del alumnado.

En otro plano, lo mismo podemos afirmar cuando la evaluación no se refiere al aprendizaje de los alumnos sino a la acción y funciones del profesorado, tanto cuando se evalúa su docencia como su investigación (dejo de lado, por tener un carácter claramente diferente, la gestión).

Olvidar, o dejar de lado, este carácter integrado solo tiene consecuencias negativas para la formación integral de los profesionales de la educación, volcados en algunas

4 Soy consciente de que esa no era la preocupación fundamental de quienes diseñaron los Indicadores; pero, a la vez, mantengo el valor que dicha información representa para los responsables de los centros educativos, al permitirles situar sus organizaciones en el contexto nacional o regional. Vid. Stufflebeam, D.L. y Shinkfield, J. (1987). Evaluación sistemática. Guía teórica y práctica. Buenos Aires: Paidós. 
funciones -tal vez por su mayor relevancia social, por el superior prestigio o hasta por cuestiones económicas- con menosprecio de otras que, también, les son atribuidas y constituyen parte de su responsabilidad.

Parece, por tanto, que los responsables del sistema deberían asumir las responsabilidades precisas para hacer de la evaluación del profesorado una tarea no solo integral, como hemos señalado, sino integrada, lo que redundaría no solo en su propio beneficio -mayor calidad de su capacitación profesional- sino en la mejora del alumnado que le ha sido encomendado.

\section{Evaluación integradora}

Y, por último, el carácter integrador de la evaluación. Si en taxonomías como la de Bloom (1981) y colaboradores la evaluación constituye el tipo de objetivos cognoscitivos más elevados y, dentro de los afectivos, se sitúa en el punto que soporta el comportamiento y el compromiso con los valores, no es por casualidad. En el primer caso, representa el hecho de la superior madurez de la mente, mientras en el segundo sitúa a la persona ante la necesidad de ser afectiva y conductualmente coherente con lo que la mente le presenta como algo bello, algo bueno, algo verdadero.

Pues bien: es este hecho el que concede a la evaluación ese especial valor integrador de la acción de los miembros de la comunidad educativa. Alumnos, profesores, padres, se ven interpelados por lo que representa una evaluación así planteada y se sentirán impelidos hacia un comportamiento coherente con los resultados.

\section{Los principios: armonía y unidad en los planteamientos}

La educación es una influencia de personas -los educadores- sobre personas -los educandos- para estimular, apoyar y fomentar el máximo perfeccionamiento de estos.

La influencia la ejercen, a lo largo del tiempo, personas que desarrollan funciones muy diferentes, en contextos muy diversos, como responsables de la enseñanza, la orientación, la tutoría...

En todos estos aspectos cada educador pretende dejar su impronta, merced a su estilo, su talante, su comportamiento, sus técnicas... fruto de su manera de ser, de su formación, de su experiencia. Y esta diversidad es buena, entre otras razones porque se ejerce sobre unos alumnos también con manifestaciones variadas de diversidad.

Sin embargo, no debemos confundir diversidad de actuaciones con planteamientos personales y profesionales que puedan llegar a ser contrapuestos entre sí. En este sentido, es recomendable que la evaluación responda a principios acordados, a pautas coherentes, a criterios consensuados... que no solo faciliten el proceso de aprendizaje a los alumnos sino que, fruto de la armonía de planteamientos, contribuya a la propia formación en tema tan relevante como es el logro de criterios propios para juzgar sobre la realidad que les rodea, en sus diversas manifestaciones: belleza, verdad o bondad, o en otros aspectos menos elevados, como la utilidad, la relevancia o la prudencia, pongamos por caso.

En efecto: comprobar que entre sus educadores se dan enfoques evaluadores coherentes ayuda a los alumnos a organizar su trabajo además de reforzar los planteamientos de estudio, pero, a la vez, les permite conocer, aplicar y asumir criterios relevantes para 
ser aplicados por ellos no solo en su vida académica sino en la profesional y la personal. Y no olvidemos que contar con criterios rectos para valorar la bondad, la verdad y la belleza, son el fundamento de la vida moral, del compromiso y caracterización por un sistema de valores, que puede contribuir a avanzar en la deseable unidad de vida.

Además de este principio de la armonía, que hemos referido fundamentalmente a la acción evaluativa que ejercen los profesores en la valoración de los aprendizajes, sería conveniente avanzar en la unidad de acción y de enfoques, de forma que la evaluación de programas, de proyectos, de materiales y recursos, del ambiente educativo, de aulas y centros, estuvieran concebidos, también, al servicio de tales principios, convenientemente matizados por las peculiaridades de cada uno de los objetos a evaluar.

En efecto: nada se opone, sino todo lo contrario, a que la tarea evaluadora tenga el carácter instrumental al que nos hemos referido en la evaluación de programas; personalmente vengo defendiendo su evaluación no solo para constatar el grado de eficacia, sino su uso al servicio de su mejora técnica o metodológica y de las personas a las que afecta, como son los profesores, que pueden encontrar en ella una base sólida para su desarrollo profesional.

Obviamente, el razonamiento es aplicable a la evaluación de centros educativos, tomados como organizaciones al servicio de un Proyecto de calidad. Si bien es verdad que los objetos a evaluar en ese ámbito son muchos, complejos y muy diversos, nada impide considerar que un centro educativo es algo así como un programa de programas; el Proyecto educativo, en efecto, requiere para su logro que los programas que en él se lleven a cabo -proyectos curriculares, planes de acogida, planes tutoriales y, por supuesto, los programas específicos de cada una de las materias y asignaturas- estén guiados por el principio de mejora para que de su armonía y coherencia de planteamientos se pueda seguir la mejora de los miembros de la comunidad educativa.

\section{Los principios: el fundamento ético de la evaluación}

Si bien en Educación no es habitual evaluar a las personas sino sus actos, sus actividades, sus comportamientos, sus logros, no es menos cierto que de la evaluación se siguen consecuencias muy relevantes para las personas individual o grupalmente consideradas. No hay más que ver cómo el profesorado se ha resistido a su evaluación por parte de los alumnos para comprender que en los evaluados siempre surge un temor ante el hecho evaluador. Este temor, más o menos fundado, tiene en su base elementos razonables: el posible fracaso, el estigma del suspenso, el qué dirán los compañeros...

Y detrás de ello, muchas veces están las carencias del proceso y del procedimiento evaluador, en particular del objeto realmente evaluado (pocas veces estamos ante pruebas auténticamente criteriales que presenten un dominio perfectamente definido), de los criterios de evaluación de los evaluadores e, incluso, del hecho de que un mismo criterio pueda ser aplicado de forma diferente por los distintos evaluadores.

Pero hay, más allá de lo anterior, aunque sin oponerse a ello, un temor que puede incluso tener algo de irracional, al no basarse en la constatación de hechos y razones objetivas, pero sí en experiencias compartidas. Es el temor al juicio técnico de quien evalúa como si sus criterios fueran cuasi dogmas, sin que le pase por la cabeza la posibilidad de enfoques diferentes. Podemos poner por ejemplo el enjuiciamiento 
de producciones científicas desde perspectivas paradigmáticas no compartidas o de currículos personales desde enfoques propios de escuelas de pensamiento diferentes.

Parece obvio que, en estos casos, sabiendo que no estamos ante dogmas, nuestro criterio técnico, tal como pide Bloom (1981) hablando de los objetivos de síntesis y evaluación, no debería centrarse en la opción elegida por las personas que presentan ese trabajo sino en la coherencia interna de los planteamientos, dando aquel por bueno en el sentido de no valorable a los efectos de su calidad.

No es la primera vez que me he visto ante valoraciones negativas de proyectos de investigación en el marco del paradigma cuantitativo realizadas por evaluadores adscritos al paradigma cualitativo -y viceversa- sin analizar que, al margen de este posicionamiento inicial, una opción legítima, el trabajo era de calidad, internamente coherente y respondiendo a los estándares propios del paradigma seleccionado.

Y no es la primera vez en que el curriculum vitae de aspirantes a profesores numerarios de universidad ha sido objeto de rechazo por tribunales mayoritariamente adscritos a escuelas didácticas, psicológicas o investigadoras diferentes a las de los candidatos, en ocasiones como si de una guerra se tratara para ganar relevancia y mayor visibilidad para ciertas doctrinas en detrimento de sus rivales.

Y todavía cabe una situación más preocupante, en este caso desde una perspectiva muy diferente, que es la de personas cuyos juicios de valor se acomodan a las circunstancias, como puede ser el medio en el que evalúan o los resultados esperados por quienes les contratan.

No es menor el problema ético que puede darse en la certificación y acreditación de personas y de organizaciones. No es raro el caso en que personas carentes de la formación necesaria se embarcan en procesos y procedimientos complejos cuya naturaleza y componentes no dominan, pero que asumen por razones de prestigio o economía. Ahora bien: es difícil, por no decir imposible, poder juzgar correctamente algo cuya naturaleza se desconoce con la debida profundidad y hacerlo sin el dominio de la metodología y de los criterios adecuados.

Sin embargo, es cierto que no disponemos de tantos evaluadores capacitados para llevar a cabo tantas y tan complejas evaluaciones como las que se realizan en nuestro país en el ámbito de la Universidad (planes de estudio, acreditaciones) o en los niveles previos a esta. Si esta afirmación es cierta, y tengo motivos para mantenerla, la consecuencia parece clara: muchas evaluaciones no respetan ciertos principios éticos, como pueden ser los recogidos en los Estándares para la evaluación de programas.

Ahora bien: el planteamiento crítico no se refiere solo a los evaluadores; alcanza también a los evaluados sean estas personas u organizaciones (Pérez Juste, 2006). Desde el falseamiento de pruebas y trabajos (que demanda de los evaluadores la correspondiente alerta y preparación para detectarlo) a la puesta en marcha de procesos de certificación de centros, desconocidos por el profesorado y con solo la decisión de los equipos directivos, el campo de actuaciones al margen de la ética es muy amplio.

Y ello sin entrar en situaciones en las que lo que se pervierte es el objetivo: lograr la acreditación/ certificación en lugar de alcanzar la calidad para, después, poder certificarla.

Y no se resuelve el problema, como a veces se pretende, planteando la atribución apriorística de valores a los aspectos a evaluar con cierto detalle y hasta con minuciosi- 
dad, queriendo dar la impresión de una máxima objetividad. En efecto: con frecuencia se construyen baremos en los que cada ítem tiene -sin más- un peso prefijado. Si la situación se lleva al extremo, el acto evaluador podría realizarlo una persona de la Administración que simplemente sepa sumar y restar. El problema es la valoración de la calidad de cada ítem y no el ítem mismo y la ponderación inicialmente atribuida, y aquí es donde surgen las discrepancias, para las que lo único que se debe esperar, y exigir, es que cada evaluador sea honesto en la atribución del valor.

\section{Acciones al servicio de estos principios}

Probablemente, ninguna acción más relevante y urgente que la formación y capacitación de todas aquellas personas que, en el ámbito educativo, desempeñan funciones evaluadoras, bien como profesionales de la evaluación (expertos en la evaluación del aprendizaje, de los programas y las organizaciones, e, incluso, del propio sistema) bien como profesionales de la educación que se sirven de la evaluación para alcanzar sus objetivos (evaluación formativa, tanto procesual como diagnóstica) o para cumplir con las exigencias de la sociedad (evaluación sumativa).

Es más, y desde otra perspectiva y ya en el contexto de la enseñanza / aprendizaje, la capacitación del propio alumnado para llevar a cabo tanto la valoración de su propio aprendizaje como el de sus compañeros, merced a la asimilación de criterios relevantes y a la práctica en la utilización de los mismos.

La evaluación es una actividad compleja y difícil para la que, en general, apenas se forma a los educadores. Este hecho, lamentable, tiene consecuencias de todo tipo, algunas tan pintorescas como las derivadas de afirmar que el criterio de evaluación de una asignatura es "sacar un 5" en lugar de indicar qué es preciso demostrar para merecer tal calificación; o indicar a los alumnos que cuando alcancen $X$ puntos en un trimestre o curso la asignatura está superada, obviando que en una situación como esta, muchos alumnos no se preocuparán de seguir aprendiendo porque lo que les importa fundamentalmente es la calificación y no el saber que la calificación acredita. Estas expresiones, y muchas otras del mismo tipo y nivel, son muy conocidas por cualquier persona que haya dedicado atención al tópico de la evaluación y nos sitúan ante un hacer evaluador alejado de todo valor formativo y, lo que es peor, dando lugar a efectos no planeados, no deseados y hasta contraproducentes.

Ya hace tiempo planteaba esta necesidad de formación en mis reflexiones sobre la evaluación (Pérez Juste, 2006):

- La necesidad de una formación básica de los docentes en esta importante tarea, para que sean capaces de llevarla a cabo con la necesaria calidad.

- La elaboración de modelos factibles, sencillos y asequibles, que no disuadan al profesorado de su aplicación y que no les distraigan de su fundamental función: la educación de sus alumnos.

En este momento, considero que tales planteamientos deberían ser tomados en consideración tanto en el marco de la formación inicial de profesores y como en el de la formación permanente. 


\section{Formación/capacitación pedagógica}

Si bien cabría plantearse de inmediato la necesidad de una capacitación técnica, prefiero comenzar por algo previo, más de fondo. No deja de ser un error acometer la evaluación en aulas y escuelas sin un conocimiento claro, preciso y, al menos, suficiente, sobre el hecho educativo que, no lo olvidemos, es la realidad a la que como mero instrumento sirve la evaluación.

Así, el desconocimiento por parte de los profesores de ese carácter instrumental, de las características evolutivas del alumnado a evaluar, de las peculiaridades de cada campo del saber académico o de las consecuencias de la evaluación para la formación integral del propio alumnado puede conducirles a cometer errores de bulto con consecuencias graves para alcanzar la meta de una personalidad sólida, estable, con un sentimiento de valía y de autoestima suficiente. Es más: tal desconocimiento privará al propio profesorado de una excelente a la vez que relevante fuente de información para su mejora y desarrollo profesional.

No es menos importante la necesidad que tiene el profesorado de situar sus responsabilidades al frente de las asignaturas y de la evaluación del correspondiente aprendizaje en un contexto más profundo, amplio y relevante: el de la formación integral de su alumnado, planteada como meta del proyecto educativo, al que todas las asignaturas deben contribuir en armonía en lugar de enfocarse como si cada una de ellas tuviera sentido por sí misma.

En este sentido, los profesores deben conocer el efecto motivador y desmotivador de las calificaciones y su incidencia sobre la satisfacción de las necesidades básicas, sea en línea con la propuesta de $\mathrm{Maslow}^{5}$ o en la menos conocida, pero interesante desde la perspectiva pedagógica de M. Yela ${ }^{6}$ o de V. García Hoz cuando se refiere a los sentimientos psicológicos básicos ${ }^{7}$.

Y, desde luego, el conocimiento del uso adecuado de la información tanto en el ámbito de las sesiones de evaluación, donde se ofrece una visión amplia, aunque no siempre integrada del progreso del alumnado, como en el privilegiado de la relación tutorial.

Esta formación debe alcanzar aspectos tan relevantes como la ayuda al educando, a través de la evaluación, para su autoconocimiento, para su autoaceptación, para facilitar el tránsito de una híper o hipovaloración a una autovaloración equilibrada y realista, que le permita asumir aquellos retos, exigentes, pero al alcance de sus posibilidades poniendo a su servicio los niveles de esfuerzo precisos.

\section{Formación/capacitación técnica}

Al igual que para muchos profesores se da una equiparación entre temario y programa, entre enseñar y explicar, entre aconsejar y tutorizar, en el caso de la evaluación el profesorado tiene con demasiada frecuencia la idea de que evaluar es, simplemente,

5 Fisiológicas, de seguridad, de afiliación, de reconocimiento y de autorrealización.

6 Valer para algo, valer para alguien, valer algo; además, una necesidad radical de aumentar el ámbito de sus necesidades.

7 Seguridad, dignidad, solidaridad. 
preguntar o proponer tareas y calificar las respuestas. Las investigaciones al respecto han sido múltiples desde hace décadas y los resultados, negativos, coincidentes (Bonboir, 1974, De la Orden, 1982).

Sin embargo, hay aspectos fundamentales a los que no suele concederse la importancia que requieren, comenzando por el diseño de tales pruebas y de las actividades y trabajos de mayor o menor amplitud y envergadura utilizados en la evaluación. Destacamos entre ellos, en efecto, el propio diseño, destinado a asegurar la fiabilidad de los resultados, íntimamente ligada a la validez de, al menos, su contenido. En este punto, aspectos técnicos como las técnicas a utilizar y los instrumentos concretos a aplicar son fundamentales para una evaluación de calidad. En efecto; de todos es conocido que no existe la técnica de evaluación del aprendizaje que pudiéramos denominar perfecta; al contrario: cada tipología de objetivos se muestra más propicia al uso de técnicas específicas. Así, una prueba objetiva es especialmente adecuada a conocimientos fundamentales concretos y no discutibles, mientras los problemas son más adecuados para evaluar el razonamiento o las pruebas de ensayo para conocer el grado de asimilación de los saberes estudiados; del mismo modo, los tradicionales trabajos se prestan en mayor medida para la evaluación del grado de madurez alcanzado, de las capacidades de iniciativa, originalidad e innovación y, si se realizan en grupo, para el logro de competencias propias del trabajo en equipo.

Tras el diseño, seguimos con la asignación de valores a los diferentes componentes de tales objetos de evaluación; con frecuencia, y sin demasiada reflexión, cada componente recibe un valor igual al de los demás, y, cuando no es así, no se aprecia argumentación que justifique las diferencias de ponderación para los diferentes ítems y aspectos. La asignación de ponderaciones a los componentes de la evaluación debe tener coherencia con la diferente relevancia que tales aspectos tienen en el currículo académico y, por consiguiente, en la propia acción metodológica del profesorado (Pérez Juste, 1996); además, para bien, estos hechos deberían ser públicos y suficientemente conocidos desde el inicio no solo por el profesor, para asegurar su coherencia metodológica, sino por los propios estudiantes, a fin de guiar su trabajo en la dirección adecuada.

Y finalicemos con el uso de la información y la interpretación de la misma. No es infrecuente, sino todo lo contrario, que la interpretación quede limitada al apto / no apto, con la consiguiente promoción o no, en función de haber alcanzado la calificación mínima -por lo general de 5- y que la información quede reducida a su reflejo en documentos administrativos, desde las actas a los boletines de notas para el propio alumnado y su familia.

Como sabemos, estos comportamientos representan la pérdida de las grandes aportaciones de la evaluación para la mejora de todas las partes interesadas y afectadas, incluyendo, desde luego, al propio profesional de la docencia.

Si bien es cierto que este tipo de interpretaciones y usos tiene su sentido y utilidad en el contexto de las evaluaciones sumativas que todo profesional está obligado a realizar por exigencias de la sociedad, no lo es menos que en estas funciones no ejerce como auténtico profesor, esto es, no se mueve por lo que debe caracterizar a un profesional que he definido como "de ayuda" (Pérez Juste, 2012), en la medida en que la esencia de su profesión es ponerse al servicio del protagonista, el alumno, para 
que logre los objetivos y mejore de forma constante; al contrario: su actuación evaluadora es más la propia de quien, simplemente, juzga el valor o calidad de lo evaluado.

La evaluación asume esta esencial función de ayuda a la mejora cuando de ella se deriva información valiosa para la mejora del aprendizaje y de la enseñanza, del alumno y del profesor, del aula y de la organización educativa. Y todo ello implica algo más que conocer el resultado final en el formato que sea (calificaciones numéricas o no, informe valorativo...); el análisis de estos resultados o, mejor, de los aspectos o ítems que conducen al mismo, y, desde luego, el conocimiento de los procesos desarrollados, se nos antojan esenciales para que evaluación responda a su esencia, que hemos situado en la mejora.

Si, como afirma Hills (1982), nadie ha aprendido nada de una calificación, no podríamos afirmar lo mismo de un informe evaluativo formativo en el que queden de manifiesto, de forma breve pero precisa, en algo parecido a un DAFO, los aspectos positivos y negativos, las carencias y las limitaciones, los errores, pero también los aciertos, con su gran valor motivador. Investigaciones como la ya añeja de Page (1958) o las más cercanas recogidas por Bonboir (1974) lo ponen de manifiesto.

Y tal valor alcanza niveles elevadísimos si el informe -y esto sería mérito de una adecuada planificación y diseño de las pruebas- es capaz de detectar la naturaleza de las causas que hay detrás de los aspectos negativos y de los mejorables.

Por último, para que el círculo de la mejora se cierre en forma virtuosa, nada mejor que asociar a los propios alumnos a las decisiones de mejora, haciéndoles protagonistas de las mismas tanto para ellos como para sus compañeros, en una especie de tutoría mutua, según las indicaciones de Bloom.

\section{Formación/capacitación ética}

Como ha quedado de manifiesto en otro momento de este documento, la evaluación debe ir asociada a un fuerte componente ético.

Por su propia naturaleza, la evaluación, que no es en su esencia sino un juicio de valor, está reservada en su plenitud a la persona con la debida capacitación. El evaluador puede ser equiparado en parte a la función arbitral, que no puede ejercer cualquiera sino la persona formada y capacitada.

Si aun así los profesionales que ejercen en algún momento funciones de juicio -como los jueces, los árbitros deportivos, los periodistas, los críticos literarios- pueden cometer $\mathrm{y}$, de hecho, cometen errores incluso graves, debemos tomar conciencia de las precauciones que deben asumir los evaluadores en el campo de la educación.

Me niego a admitir, aunque puede que los haya, evaluadores como los citados que ejerzan su función mal a sabiendas; hablamos de la prevaricación de un juez, del árbitro deportivo que se vende por dinero, del periodista que, contra todo código ético profesional, sesga y desfigura su información para perjudicar a una parte en beneficio de otra. Pero también entre nosotros cabe pensar en los miembros de los tribunales que emiten juicios acomodaticios en función de las personas valoradas, de la amistad o el trato con ellas, de la presión ambiental, etc.

Sin embargo, dejando de lado estos hechos por lo burdos que son, sí quiero señalar la falta de ética que puede darse cuando un evaluador es capaz de emitir una valoración 
sobre algo que no conoce con la suficiente amplitud y profundidad y cuando, sin la información necesaria sobre el contexto de la realidad evaluada, emite su juicio con hasta cierta despreocupación por las consecuencias para las partes interesadas y afectadas.

También podemos, y debemos, señalar como alejados de planteamientos éticos aquellos juicios de valor en los que se pretende conseguir objetivos que nada tienen que ver con la realidad evaluada. No es inhabitual que los profesores asignen calificaciones bajas en sus asignaturas a aquellos alumnos cuyos comportamientos dejan que desear, y ello a modo de castigo para producir un cambio en los comportamientos. Habría que preguntar a estos profesores que pueden llegar a suspender a un alumno pongamos en Matemáticas o Lengua -aunque las pruebas evidencien niveles suficientes de logro- como consecuencia de un mal comportamiento o de una actitud inadecuada, si para obtener una buena calificación deberán volver a demostrar que saben aquellas asignaturas o si han modificado el comportamiento o la actitud. Pero el problema es que tanto uno como otra pueden alterarse, pueden fingirse, a los solos fines de lograr la superación de aquella sin que en realidad las actitudes hayan sido en verdad modificadas.

Es más: ante lo percibido como injusto por el alumno, la actitud puede verse radicalizada.

En definitiva, el comportamiento ético del profesor es un componente íntimamente ligado a todo su hacer profesional y, en particular, a su función evaluadora, teniendo en cuenta las consecuencias que se derivan de esta para la formación y el progreso de sus alumnos.

\section{Capacitación de los líderes}

En el ámbito de las organizaciones educativas, de los centros, el liderazgo se ha configurado como uno de los factores esenciales que contribuyen a su buena marcha, al logro de sus objetivos que, en el caso de los centros, no deberían ser otros que los planteados en los proyectos educativos.

Si los estudios sobre las escuelas eficaces ya resaltaron con reiteración la importancia del liderazgo (Edmons, 1982; Scheerens, 1992; Sammons, Hilman y Montimore, 1995) y si los modelos de calidad, desde Deming o Baldrige a Gento (1994), pasando por ISO o EFQM, todos recogen este factor, es porque sus funciones resultan imprescindibles para la creación de actitudes favorables hacia la calidad y, en particular, para hacer de los centros verdaderas organizaciones, organizaciones que aprenden y crean y gestionan conocimiento (Gairín, 2009), en las que la evaluación cobre una relevancia especial.

Pensemos en tareas y responsabilidades tan importantes como son las que van desde los acuerdos entre profesores en materia de planteamientos técnicos sobre la evaluación de los aprendizajes a la promoción y estímulo de la evaluación de la propia organización, pasando por la creación de la cultura de la evaluación en el ámbito de los programas y a la búsqueda de la coherencia y la armonía a las que ya hemos hecho alusión al hablar de los principios y criterios. En todas estas tareas y funciones, la iniciativa, el compromiso y el protagonismo del líder son fundamentales para que el centro y su personal avancen en la dirección adecuada y perseveren en el intento. 


\section{El valor de la autoevaluación}

Si pedagógicamente hablando el sentido y naturaleza de la evaluación no es otro que la mejora, no podemos dejar de lado el valor de la autoevaluación tanto para el perfeccionamiento personal y profesional como para la formación integral.

Comenzando por esta última, debemos destacar la importancia que representa el hecho de alcanzar los objetivos más elevados de la formación intelectual según taxonomías como la de Bloom y cols., y su contribución a la formación moral según planteamientos como los del propio Bloom, de Piaget o de Kohlberg ${ }^{8}$.

En efecto: si las aportaciones de los colaboradores de Bloom hacen referencia a la dimensión moral, Kohlberg, sobre la base de los avances de Piaget, propone una metodología para el desarrollo del juicio moral hasta llegar a la madurez moral -algo que muy pocas personas, parece ser, alcanzan. Tal madurez moral tiene en su base la madurez intelectual, traducida en la autonomía intelectual, soporte a su vez de la autonomía moral.

Bloom y Hasting proponen una tipología de objetivos afectivos, en parte superpuestos sobre los cognitivos, que evidencian tal relación:

- Valoración: Aceptación de un valor. Preferencia por un valor. Compromiso.

- Organización: Conceptualización de un valor. Organización de un sistema de valores.

- Caracterización por un valor o complejo de valores: Inclinación generalizada. Caracterización.

Por su parte, Kohlberg, con sus tres grandes niveles y sus seis estadios, sitúa el desarrollo del juicio moral como una de las grandes metas deseables. Pero, claro está, quienes alcanzan tal autonomía, a niveles razonables, son capaces de aplicarla a su trayectoria vital y profesional, merced a la claridad de juicio. No obstante, y este es un paso más, pero para el que la evaluación -en este caso el conocimiento y aprecio de los criterios- evidencia su limitación de mero instrumento, de mera herramienta, el paso siguiente, el del comportamiento acorde con lo intelectualmente apreciado como bueno y afectivamente valorado como tal, depende ya de la fuerza de voluntad al servicio del bien.

No obstante, no es despreciable, ni mucho menos, el servicio de la evaluación a la persona, a la que pone a las puertas de la decisión moral y más, del comportamiento moral.

Cabe reseñar que el aprendizaje por parte del alumnado puede realizarse en paralelo a la acción evaluadora del profesorado. Y mantengo que tal aprendizaje y ejercicio del mismo es mucho más relevante que el hecho de asociar las autovaloraciones del alumno a la configuración de la calificación final, sumativa, asignándoles un determinado porcentaje.

8 Hersh, R., Reimer, J. y Paolitto, D. (1988). El crecimiento moral. De Piaget a Kohlberg. Madrid: Narcea. 


\section{Referencias}

Bloom, B.S. Hasting, J.Th. y Madaus, G.F. (1981). Evaluación del aprendizaje. Buenos Aires: Troquel.

Bonboir, A. (1976). La docimología. Problemática de la evaluación. Madrid: Morata.

Comité Conjunto de Estándares para la Evaluación Educativa (1998) Estándares para la evaluación de programas. Bilbao: Ediciones Mensajero.

De la Orden Hoz, A. y González Soler, A. (1982). Análisis de las pruebas finales de EGB. Revista Española de Pedagogía, 156, 7-40.

De la Orden Hoz, A. (1988). La calidad de los centros educativos, asunto para un congreso, Bordón, 40(2), 149-162.

Edmons, R. (1982). Programs of Schools Improvement: An Overview, Educational Leadership, 40(3), 4-11.

Escudero Escorza, T. (1980). ¿Se pueden evaluar los centros educativos y sus profesores?. I.C.E. de la Universidad de Zaragoza: Zaragoza.

Garín, J. y otros (2009). Estadios organizativos y gestión del conocimiento en instituciones educativas. Revista de Ciencias Sociales. 15(4), 620-634.

Gento Palacios, S. (1994). La gestión de calidad en Educación. Madrid: La Muralla.

Id. (1998). Implantación de la calidad total en las instituciones educativas. Madrid: UNED.

Hills, J.R. (1982). Evaluación y medición en la Escuela. México: Kapelusz Mexicana.

Joint Committée on Standards for Educational Evaluation (1994). The Program Evaluation Standards. Berverly Hills: Sage Pub.

Luján, J. y Puente, J. (1996). Evaluación de Centros docentes. El plan EVA. Madrid: MEC.

Ministerio de Educación y Ciencia (varios años) Sistema Estatal de Indicadores de la Educación. Recuperado de http://www.mecd.gob.es/inee/sistema-indicadores.html.

Ministerio de Educación y Ciencia. Instituto de Evaluación. (2015). PISA 2015 Programa para la Evaluación Internacional de Alumnos de la OCDE. Informe Español. Madrid: Autor. Recuperado de http://www.mecd.gob.es/inee/estudios/pisa.html.

Nevo, D. (1986). The Conceptualization of Educational Evaluation. En E. R. House (Edit.). New Directions in Educational Evaluation (pp.15-30). Lewes: Falmer Press.

Page, E.B. (1958). A seventy-four classroom experiment in school motivation. Journal of Educational Psychology, 49, 173-181.

Pérez Juste, R. y García Ramos, J.M. (1989). Diagnóstico, evaluación y toma de decisiones. Madrid: Rialp.

Pérez Juste, R. (1996). La evaluación en la Universidad. En V. García Hoz (Dir.) La Educación personalizada en la Universidad: Tratado de Educación Personalizada (Vol. 27, pp. 431-478). Madrid: Rialp.

Pérez Juste, R. (2006). Evaluación de Programas Educativos. Madrid: La Muralla.

Pérez Juste, R. (2007). Quality Education: Educational Personalization and Social Pertinence, En J.E. Benton y P. Swami: Creating Cultures of Peace. Pedagogical Thounght and Practice (pp. 29-44). San Diego (EEUU): World Council for Curriculum and Instruction.

Pérez Juste, R. (Coord.) (2012). El Portfolio. Aprendizaje, competencias y evaluación. Madrid: UNED.

Sammons, P., Hillman, J. y Mortimore, P. (1995). Key characteristics of effective schools: a review of school effectiveness research. London: OFSED and Institute of Education. 
Scheerens, J. (1992). Effective Schooling Research. Theory and Practice. Londres: Casell. UNESCO (1998). Declaración Mundial sobre la Educación Superior en el siglo XXI: Visión y Acción. UNESCO. Recuperado de http://www.unesco.org/education/educprog/ wche/declaration_spa.htm.

Yarbrough, D.B. y otros (2011). Las normas de evaluación de programas: Una guía para los evaluadores y los usuarios de la evaluación (3ª Ed.). Thousand Oaks, CA: Sage.

Fecha de recepción: 13/10/2015

Fecha de revisión: 15/10/2015

Fecha de aceptación: 13/11/2015 